\title{
Micro-CT evaluation of the radioprotective effect of resveratrol on the mandibular incisors of irradiated rats
}

\section{Gabriella Lopes DE REZENDE BARBOSA(a) $^{(a)}$ \\ Luiz André PIMENTA(b) \\ Solange Maria de ALMEIDA(c)}

(a) Universidade de Campinas - Unicamp, Piracicaba Dental School, Department of Oral Diagnosis, Piracicaba, SP, Brazil.

(b) University of North Carolina at Chapel Hill, School of Dentistry, Department of Dental Ecology, Chapel Hill, NC, USA.

(c)Universidade de Campinas - Unicamp, Piracicaba Dental School, Department of Oral Diagnosis, Piracicaba, SP, Brazil.

Declaration of Interests: The authors certify that they have no commercial or associative interest that represents a conflict of interest in connection with the manuscript.

Corresponding Author: Gabriella Lopes de Rezende Barbosa Email: gabriellalopes@live.com

DOI: 10.1590/1807-3107BOR-2016.vol30.0168

Submitted: Apr 17, 2015

Accepted for publication: Nov 02, 2015

Last revision: Dec 14, 2015

\begin{abstract}
The purpose of this study was to perform a microcomputed tomographic evaluation of the radioprotective effect of resveratrol on the volume of mandibular incisors of irradiated rats. A second aim was to make a quantitative assessment of the effect of $x$-ray exposure on these dental tissues. Twenty adult male rats were divided into four groups: control, irradiated control, resveratrol, and irradiated resveratrol. The resveratrol groups received $100 \mathrm{mg} / \mathrm{kg}$ of resveratrol, whereas the irradiated groups were exposed to 15 Gy of irradiation. The animals were sacrificed 30 days after the irradiation procedure, and their mandibles were removed and scanned in a microcomputed tomography unit. The images were loaded into Mimics software to allow segmentation of the mandibular incisor and assessment of its volume. The results were compared by One-way ANOVA and Tukey's post hoc test, considering a 5\% significance level. The irradiated groups showed significantly diminished volumes of the evaluated teeth, as compared with the control group $(p<0.05)$. The resveratrol group presented higher values than those of the irradiated groups, and volumes similar to those of the control group. High radiation doses significantly affected tooth formation, resulting in alterations in the dental structure, and thus lower volumes. Moreover, resveratrol showed no effective radioprotective impact on dental tissues. Future studies are needed to evaluate different concentrations of this substance, in an endeavor to verify its potential as a radioprotector for these dental tissues.
\end{abstract}

Keywords: Odontogenesis; Radiation-Protective Agents; Radiation Injuries; X-Ray Microtomography.

\section{Introduction}

Radiation treatment, surgical procedures and chemotherapy are the main treatments for neoplasms of the head and neck region. ${ }^{1}$ In the case of radiotherapy, the result of these treatments is the formation of free radicals, leading to ionization and deleterious effects on the cells of many normal, uninvolved tissues. Examples of structures that receive significant doses of radiation include the salivary glands, the oral mucosa and the teeth.

Changes in odontogenesis may occur in patients undergoing head and neck radiotherapy during tooth formation, such as children with neoplastic diseases. ${ }^{2}$ These alterations include enamel hypoplasia, interference in enamel organ development and tooth formation, in addition to microdontia 
and changes in root formation, such as interruption, thinning and enlargement of the pulp chamber. The severity of the damage depends on the stage of dental development, as well as the dose and extension of the irradiated area. ${ }^{3}$ Prior studies have reported the effects of high radiation doses on rat incisors, such as delayed eruption and disturbances in secretory functions ${ }^{4,5}$ However, few studies have quantitatively assessed the damage caused by irradiation to dental tissues during formation.

Radioprotective substances have been developed to protect against or minimize these unwanted deleterious effects on healthy tissues, and have become an integral part of patient care and enhancement of quality of life. ${ }^{6,7}$ These substances should not only have antioxidant properties, but also have anti-inflammatory and antimicrobial properties, be immunostimulants, stimulate cell proliferation, and not cause systemic toxicity. ${ }^{8}$

Different substances tested as radioprotectors include organic substances, such as resveratrol (3,5, ' $^{\prime}$-trihydroxy-trans-stilbene), which is a polyphenol belonging to the group of compounds called phytoalexins. ${ }^{9}$ This substance is synthesized by plants when stimulated by microbial infection, ultraviolet radiation, and ozone exposure. ${ }^{10}$ Grapevines (Vitis vinifera and Vitis labrusca) are among the species considered as having a high potential for synthesis of the compound. ${ }^{11}$

Attention and interest in this substance have increased following evidence that resveratrol can prevent against, or at least delay, the onset of cancer, ischemic heart disease, chemically induced injury, diabetes, pathological inflammation and viral infection. ${ }^{12}$ Studies have been conducted regarding its radioprotective effect, and have shown positive effects, including the radioprotection of glandular and bone marrow cells. ${ }^{13,14}$

The effects caused by substances of different origin and radiation can be evaluated by several different methods, depending on the tissue of interest and the expected outcome. Currently, whole structures can be assessed in micrometer scale without sample destruction, owing to new imaging techniques, such as microcomputed tomography $(\mu \mathrm{CT})$. The use of $\mu \mathrm{CT}$ allows the accurate appraisal of mineralized structures, as well as their volume, morphology and internal structure. ${ }^{15}$

Therefore, the aim of the study was to make a $\mu \mathrm{CT}$ evaluation of the radioprotective effect of resveratrol on the volume of mandibular incisors of irradiated rats, and also make a quantitative assessment of the impact of x-ray exposure on dental tissues.

\section{Methodology}

After receiving approval from the Animal Research Ethics Committee (CEUA/UNICAMP, protocol no. 2317-1), twenty 8-week-old adult male rats (Rattus novergicus albinus, Wistar) were obtained for the study. The animals were kept on a diet of food and water ad libitum, at $23^{\circ} \mathrm{C}$ room temperature and artificial lighting (light-dark cycles of 12 hours). The study was carried out in accordance with EU Directive 2010/63/EU. The animals were randomly divided into four groups $(\mathrm{n}=5)$ : control (received no medication and no irradiation), irradiated control (received a single dose of $15 \mathrm{~Gy}$ irradiation), resveratrol (received resveratrol solution) and irradiated resveratrol (received resveratrol solution and $15 \mathrm{~Gy}$ irradiation). The sample size selection was based on the "resource equation" method. According to this method, an "E" value is measured, representing a degree of freedom of analysis of variance, and should lie between 10 and 20. E can be measured by subtracting the total number of animals from the total number of groups. Thus, any sample size that keeps E between 10 and 20 should be considered as adequate. The present study had an E value of $16 .^{16}$

Resveratrol was prepared by diluting this compound in a $100 \%$ ethanol solution to a concentration of $50 \mathrm{~mL} / \mathrm{kg}$. The resveratrol/ethanol solution was then diluted in distilled water as follows: $0.05 \mathrm{~mL}$ of resveratrol $(50 \mathrm{mg} / \mathrm{mL})$ in $0.2 \mathrm{~mL}$ of distilled water to a final concentration of $10 \mathrm{mg} / \mathrm{mL}$.

The medication was administered via enteral feeding, using a plastic catheter connected to a $3.0 \mathrm{~mL}$ syringe, to allow direct deposition into the stomach. Each rat received a dose of $100 \mathrm{mg} / \mathrm{kg}$ resveratrol, administered by gavage once every 24 hours for 2 days before irradiation, and again on the day of irradiation, 30 minutes before exposure to radiation. ${ }^{14}$ All the solutions were prepared immediately before 
the experiments, kept in amber glass bottles, protected from light, and stored at room temperature.

All the animals undergoing irradiation were weighed and anesthetized by intramuscular administration of ketamine chloride and xylazine chloride solutions. Once anesthetized, the animals were placed on a linear accelerator with $18 \times 30 \mathrm{~cm}$ collimation, and only the head and neck region was exposed to a single dose of 15 Gy x-ray radiation. After 30 days, all the animals were sacrificed and the mandibles were removed. The specimens were formalin-fixed for further examination.

The left hemi-mandibles were scanned by a microcomputed tomography scanner (SkyScan 1174, Kontich, Belgium), operating at $50 \mathrm{kVp}$ and $800 \mu \mathrm{A}$, with a voxel size of $22.5 \mu \mathrm{m}, 0.45$ degrees rotation steps and 360 degrees rotation, using a $0.5 \mathrm{~mm}$ aluminum filter. The entire incisor was included in the field of view, and the images were reconstructed after acquisition, using NRecon software (SkyScan, Kontich, Belgium).

The reconstructions of the tooth images were imported into the Mimics software program (version 16.0, Materialise Medical, Leuven, Belgium), segmented and analyzed by a single well-trained radiologist. The threshold was defined so as to include the teeth but remove any visible extraneous scatter, artifacts, and background. The volumetric region of interest (VOI) was cropped and narrowed to include only the region of the incisor comprised between the distal surface of the second molar and the digastric muscle insertion to the mandible (Figure 1).

After defining the boundaries, a combination of manual slice and threedimensional editing was used to obtain refined surfaces of the tooth segmentation. The resulting set of masks of the VOI was rendered into a shaded surface mesh by the same software program, and each segmented volume was calculated, according to the manufacturer's recommendations.

After a one-month interval, half of the sample was reassessed to calculate the intraobserver reproducibility by Intraclass correlation coefficient, using MedCalc software, version 11.2.1.0 (MedCalc Software, Mariakerke, Belgium). One-way analysis of variance (ANOVA) was used to determine the overall differences among the volumes of the four groups. The test was performed using BioEstat software, version 5.0 (Instituto Mamirauá, Belém, Brazil); $p$ values $<0.05$ were considered statistically significant.

\section{Results}

The intraobserver reliability calculated by the Intraclass correlation coefficient yielded a value of 0.96 , an excellent agreement rate according to ICC interpretation.

One-way ANOVA showed statistically significant overall differences. Tukey's test was the chosen post
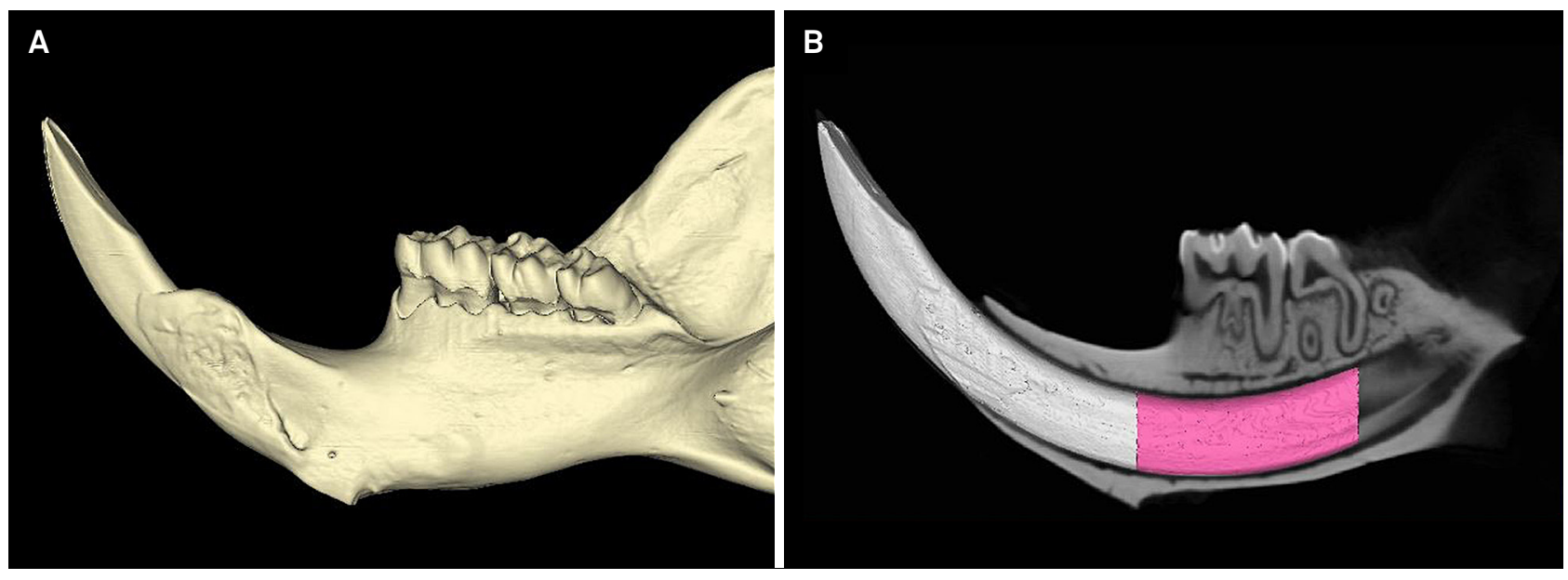

Figure 1. Three-dimensional reconstruction of the entire mandible $(A)$ and segmentation of the mandibular incisor (B) with the highlighted volumetric region of interest comprised between the second molar distal surface and the digastric muscle insertion to the mandible (pink region). 
hoc test to confirm where the differences occurred between the groups.

Significant differences $(p<0.05)$ were found in comparing the control group with the irradiated control and the irradiated resveratrol groups, as well as the resveratrol group with the irradiated control and the irradiated resveratrol groups, evidencing the impact of $x$-ray exposure on dental tissues. The lowest volume of the incisor region was observed in the irradiated control group (Table 1). No significant differences $(p>0.05)$ were detected when the irradiated control and the irradiated resveratrol groups were compared, demonstrating a lack of resveratrol radioprotection to the teeth (Table 1).

The three-dimensional reconstructions (Figure 2) clearly show the volume difference among the groups and the conformation of the incisors. The irradiated control and the irradiated resveratrol groups presented tooth defects, as well as diminished size.

\section{Discussion}

Patients under radiotherapy treatment are vulnerable to harmful side effects, such as disturbances that can impact the development of teeth. Tooth development begins in the embryonic phase but is only complete after the first decade of life. During their developmental stages, the teeth are more susceptible to environmental disturbances, such as radiation, which can affect their normal development. ${ }^{17}$ In the present study, the mandibular incisor of the rat was chosen, because of its continued growth and constant cell proliferation in the odontogenic region, replicating

Table 1.Volumetric mean values $( \pm S D)$ of the region of interest according to the evaluated groups $\left(\mathrm{mm}^{3}\right)$.

\begin{tabular}{lcc}
\hline & \multicolumn{2}{c}{ Volume } \\
\cline { 2 - 3 } & Mean & SD \\
\hline Control & $849.4^{\mathrm{A}}$ & 121.1 \\
Irradiated control & $475.0^{\mathrm{B}}$ & 137.6 \\
Resveratrol & $984.9^{\mathrm{A}}$ & 98.6 \\
Irradiated resveratrol & $539.6^{\mathrm{B}}$ & 155.7 \\
\hline
\end{tabular}

Different letters in superscript following values indicate statistical significance (level of significance set at $p<0.05$ ). the cell differentiation that occurs during tooth formation in humans.

The date chosen to sacrifice the animals was based on the continuous growth and eruption rate of the rodents' incisors. ${ }^{5}$ A period of 30 days was chosen, because it is adequate to evaluate the formation and mineralization of irradiated dental tissues. Moreover, after 30 days, the undifferentiated irradiated cells of the odontogenic region would then be positioned under the mandibular molars.

According to the present study results, radiation caused a significant defective formation of all mineralized materials composing the tooth structure. This alteration led to lower volumes of the evaluated mandibular incisor. Histological alterations in dental tissues caused by irradiation have been reported in prior literature. ${ }^{4,5,18}$ Nonetheless, the literature on the effect of these defects in the entire dental structure is scant. Mature enamel defects are important because of their relation to compromised aesthetic, masticatory functions and heightened susceptibility to caries. ${ }^{4}$

Among the large range of radioprotective substances that have been studied, resveratrol has received much attention because of its antioxidant, anti-inflammatory, analgesic, antiviral, cardioprotective and neuroprotective actions. ${ }^{19}$ However, in the present study, statistically significant differences could be observed between the irradiated control and the irradiated resveratrol groups, demonstrating that resveratrol had no radioprotective effect on dental tissues. These findings are not in agreement with the positive effects previously reported in the literature, such as the radioprotection of bone marrow cells and even of normal uninvolved tissue in the head and neck region, as described by $\mathrm{Xu}$ et al. ${ }^{13}$ and Şimşek et al. ${ }^{20}$ on the protection of salivary glands. ${ }^{14,19}$ These differences may be explained by the different types of cells that were studied, as well as the administration of the medication performed intraperitoneally in these studies.

The selected dose $(100 \mathrm{mg} / \mathrm{kg})$ is in agreement with research that found positive radioprotective effects, such as the studies by Carsten et al. ${ }^{14}$ and Şimşek et al. ${ }^{20}$ However, since no protection was 

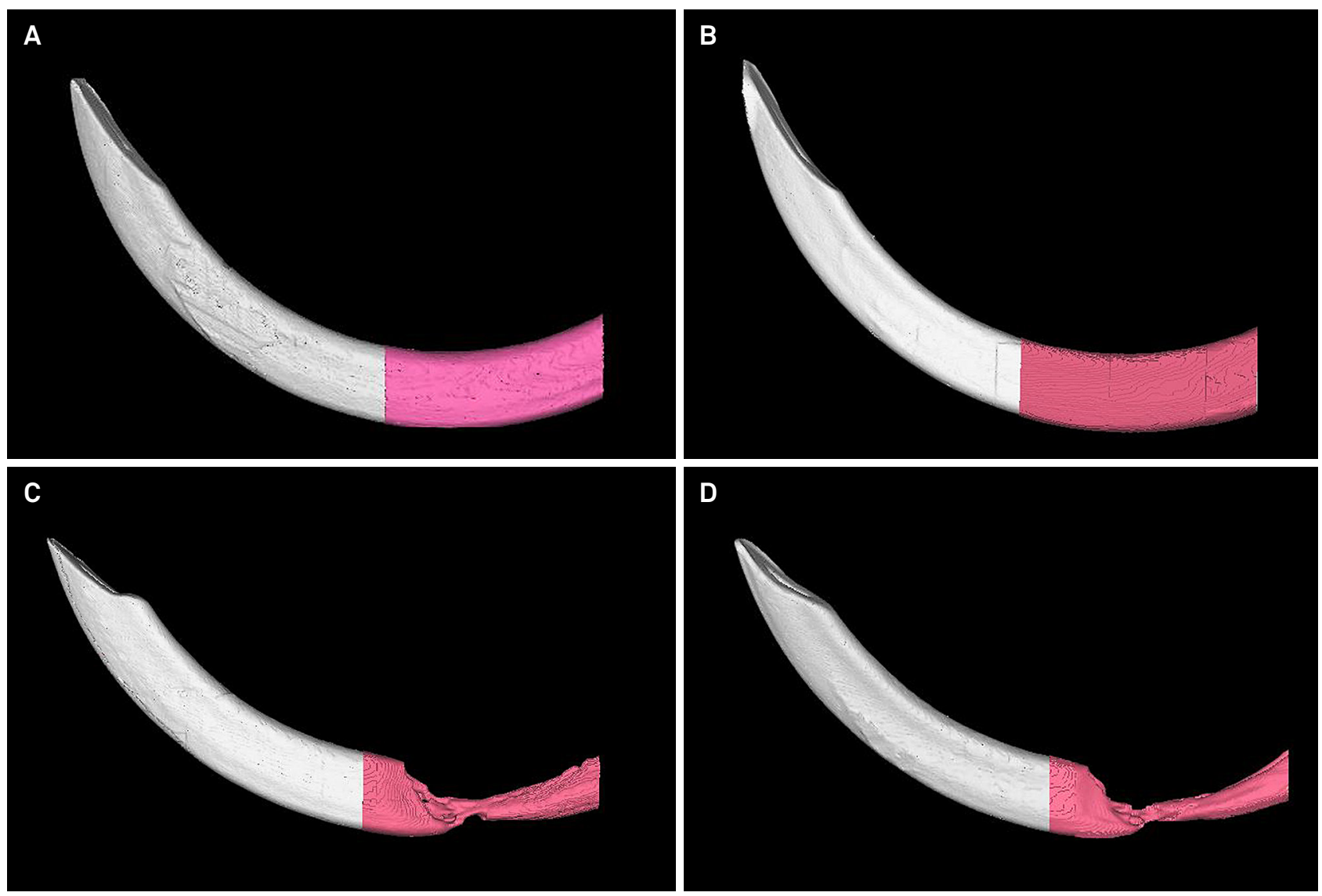

Figure 2. Three-dimensional reconstructions showing the differences in incisor volume among the groups: A- Control group, B- Resveratrol group, C- Irradiated control group, and D- Irradiated resveratrol group.

observed in the present study, this dose may not be effective in dental tissues. In this sense, further investigation is needed to evaluate different concentrations and frequency of application of this substance specifically for dental tissues, in an endeavor to prove its potential as a radioprotector.

A positive finding of the present study was that the administration of resveratrol itself showed no potential to modify dental structures, as indicated by the similar volumes of the resveratrol versus control groups $(p>0.05)$.

$\mu \mathrm{CT}$ technology was the method of choice of this study, since it provides high-resolution images and is a reliable and innovative technique that allows the assessment of volume and density of hard dental tissues, even during development. Moreover, $\mu \mathrm{CT}$ allows this assessment to be made without destroying the samples, and minimizes artifacts caused by sample preparation. ${ }^{15}$ The positive outcomes of the research corroborate those by Dong et al., ${ }^{15}$ who state that $\mu \mathrm{CT}$ technology is a feasible technique for dental tissue evaluation, because it allows a quantitative evaluation of the structure as a whole. Furthermore, this study sought to shed light on the importance of research using innovative technology and imaging modalities incorporated into animal studies, by contributing more information and performing three-dimensional views of structures and tissues without destroying the samples.

\section{Conclusion}

The results of the present study demonstrate the deleterious effect of $x$-ray radiation on tooth formation, resulting in the reduced volume of dental structures. It was also observed that the dose of resveratrol used in this study had no effective radioprotective impact on dental tissues. Future studies are needed to evaluate different concentrations of this substance in an endeavor to prove its potential as a radioprotector. 


\section{References}

1. McClure SA, Movahed R, Salama A, Ord RA. Maxillofacial metastases: a retrospective review of one institution's 15-year experience. J Oral Maxillofac Surg. 2013;71(1):178-88. doi:10.1016/j.joms.2012.04.009

2. Albuquerque RA, Morais VLL, Sobral APV. Sobral APV. [Protocol of odontological attendance of oncological pediatric patients: a literature review]. Rev Odontol UNESP. 2007;36(3):275-80. Portuguese.

3. Lopes NN, Petrilli AS, Caran EM, França CM, Chilvarquer I, Lederman H. Dental abnormalities in children submitted to antineoplastic therapy. J Dent Child (Chic). 2006;73(3):140-5.

4. Ramos-Perez FMM, Espírito Santo AR, Perez DEC, Novaes PD, Bóscolo FN, Line SRP, et al. Ionizing radiation effects on the secretory-stage ameloblasts and enamel organic extracellular matrix. Radiat Environ Biophys. 2014;53(3):589-98. doi:10.1007/s00411-014-0539-4.

5. Araujo AMM, Gomes CC, Almeida SM, Klamt CB, Novaes PD. Effect of radiotherapy on the eruption rate and morphology of the odontogenic region of rat incisors. Arch Oral Biol. 2014;59(11):1242-1248. doi:10.1016/j.archoralbio.2014.07.004.

6. Kuntić VS, Stanković MB, Vujić ZB, Brborić JS, Uskoković-Marković SM. Radioprotectors - the evergreen topic. Chem Biodivers. 2013;10(10):1791-803. doi:10.1002/cbdv.201300054.

7. Mishra S, Malhotra P, Gupta AK, Singh PK, Mishra AK, Javed S, et al. Novel method for screening of radioprotective agents providing protection to DNA ligase against gamma radiation induced damage. Int J Radiat Biol. 2014;90(2):187-92. doi:10.3109/09553002.2014.868613.

8. Arora R, Gupta D, Chawla R, Sagar R, Sharma A, Kumar R, et al. Radioprotection by plant products: present status and future prospects. Phytother Res. 2005;19(1):1-22. doi:10.1002/ptr.1605

9. Jeandet P, Douillet-Breuil AC, Bessis R, Debord S, Sbaghi M, Adrian M. Phytoalexins from the Vitaceae: biosynthesis, phytoalexin gene expression in transgenic plants, antifungal activity, and metabolism. J Agric Food Chem. 2002;50(10):2731-41. doi:10.1021/jf011429s
10. Ignatowicz E, Baer-Dubowska W. Resveratrol, a natural chemopreventive agent against degenerative diseases. Pol J Pharmacol. 2001;53(6):557-69.

11. Frémont L. Biological effects of resveratrol. Life Sci. 2000;66(8):663-73. doi:10.1016/S0024-3205(99)00410-5

12. Baur JA, Sinclair DA. Therapeutic potential of resveratrol: the in vivo evidence. Nat Rev Drug Discov. 2006 Jun;5(6):493-506. doi:10.1038/nrd2060

13. Xu L, Yang X, Cai J, Ma J, Cheng H, Zhao K, et al. Resveratrol attenuates radiation-induced salivary gland dysfunction in mice. Laryngoscope. 2013;123(11):E23-9. doi:10.1002/lary.24276

14. Carsten RE, Bachand AM, Bailey SM, Ullrich RL. Resveratrol reduces radiation-induced chromosome aberration frequencies in mouse bone marrow cells. Radiat Res. 2008;169(6):633-8. doi:10.1667/RR1190.1

15. Dong G, Dong Q, Liu Y, Lou B, Feng J, Wang K, et al. High-resolution micro-CT scanning as an innovative tool for evaluating dental hard tissue development. J Appl Clin Med Phys. 2014;15(4):335-44. doi:10.1120/jacmp.v15i4.4956

16. Charan J, Kantharia ND. How to calculate sample size in animal studies? J Pharmacol Pharmacother. 2013;4(4):303-6. doi:10.4103/0976-500X.119726

17. Nanci A. Ten Cate's Oral Histology: Development, Structure and Function. 7th ed. St. Louis: Mosby; 2007.

18. English JA, Schlack CA, Ellinger F. Oral manifestations of ionizing radiation. II. Effect of $200 \mathrm{KV}$. x-ray on rat incisor teeth when administered locally to the head in the 1,500 R. dose range. J Dent Res. 1954 Jun;33(3):377-8. doi:10.1177/00220345540330031101

19. Dobrzyńska MM. Resveratrol as promising natural radioprotector. A review. Rocz Panstw Zakl Hig. 2013;64(4):255-62.

20. Şimşek G, Gürocak S, Karadağ N, Karabulut AB, Demirtaş E, Karataş E, et al. Protective effects of resveratrol on salivary gland damage induced by total body irradiation in rats. Laryngoscope. 2012;122(12):2743-8. doi:10.1002/lary.23609 